\title{
HISTORY OF A CASE
}

IN WHICH

\section{A CEDAR PENCIL}

\section{WAS LODGED IN THE CAVITY OF THE ABDOMEN FOR EIGHT MONTHS.}

\author{
BY \\ JOHN ERICHSEN, \\ PROFESSOR OF SURGERY AT UNIVERSITY COLLEGE, AND SURGEON TO \\ UNIVERSITY COLLEGE HOSPITAL. \\ Received Nov. 18th, 1855-Rend Dec. 11th, 1855.
}

ON the 16th of May last I was requested by Mr. Bryant to see with him a young woman, 28 years of age, a governess, who had come up to London in order to have a cedar pencil, which was said to be lodged in the abdomen, extracted from that cavity. On inquiry into the history of the case, the following facts were elicited, not, however, without difficulty. The patient stated, that about the end of September, 1854, eight months before we saw her, she felt some difficulty in passing water, that, in order to relieve this, she was advised by a friend to pass a pencil into the urethra. That she attempted to do so, but, that whilst so engaged, some one happened to enter the room,-that the interruption caused the pencil to slip out of her hand, and that on sitting down shortly afterwards, she was seized with acute stabbing pain 
in the lower part of the abdomen; that the pencil disappeared, and that neither she nor the medical man by whom she was shortly examined, had been able to ascertain where it was lodged. After this, she had repeated attacks of peritonitis, but that the pencil had not been found, nor had it ever come away by any of the natural channels. She has not suffered from dysuria, or irritation of the bladder, nor has blood ever been discharged from that organ or from the vagina ; neither has there been any pain or uneasiness in defecation; blood has, however, been occasionally discharged per anum; but to this she paid no special attention, as she had for many years been the subject of piles, and occasionally had passed blood before the accident.

She now complains of constant pain in the abdomen, of a shooting or stabbing character; suffers from constant vomiting or retching, is debilitated, emaciated, and worn by her sufferings and the repeated attacks of abdominal inflammation, and is anxious to submit to any procedure that would free her from her misery.

Examination of the abdomen.-Mr. Bryant had, previously to my seeing the patient, detected what felt like the point of a pencil projecting directly forwards against the abdominal wall of the right side, about midway between the umbilicus and Poupart's ligament. On passing my hand over the abdomen, I distinctly felt this projection, apparently very superficial, as if it were just under the integument. It gave the sensation of the end of a cut pencil. It was distinctly moveable in a direction upwards, and could be pushed back somewhat, so as partly to disappear, but, on the pressure being removed, immediately returned to its former situation. The abdomen was swollen somewhat, slightly tympanitic, and uniformly tender; but more particularly so about and below the umbilicus, to which spot she referred most of her sufferings.

On passing a sound into the bladder, no trace of the foreign body could be found in the interior of that viscus, though, on turning the point of the instrument to the right 
side, a hard body could be indistinctly felt through and outside its walls.

On exploring the vagina there was no sign of the pencil in that cavity, but, on passing the finger high up, and to the right of the cervix uteri, the pencil could be felt outside and through the walls of the vagina, lying apparently directly across the body from front to back.

On examining the rectum the same conditions were found as in the vagina, viz., the foreign body could be felt high up through its walls, lying to the right of the gut. The posterior extremity seemed to be lodged in the hollow of the sacrum, but it could not be reached by the finger.

From this examination it seemed evident that the pencil lay to the outer and right side of the bladder, vagina, and rectum, stretching across the body from the point, midway between the umbilicus and Poupart's ligament in front, to the hollow of the sacrum behind, apparently directly across the cavity of the abdomen. On further examination it was found that the position of the point of the pencil projecting anteriorly could be influenced by pressing on its posterior part through the rectum, in such a way that it would seem as if it were rotating upon a central axis. Thus if the posterior part was raised, the point became depressed; if, on the contrary, the posterior part was drawn down, the anterior extremity rose upwards towards the umbilicus; if it was pushed to the right, the other moved to the left, \&c. In whatever direction, however, the anterior extremity was moved, it always came back to one fixed point,that already indicated, about two inches above Poupart's ligament.

Mr. Bryant and I, consequently, came to the conclusion, that the pencil was lying across the cavity of the abdomen, that it was fixed about its centre by some tissue traversed by it, and that the point, which felt very superficial, was engaged in the anterior abdominal wall. What was to be done under these circumstances? Was the pencil xxxix. 
to be left, or was an attempt to be made to extract it? These questions were anxiously discussed by us. If it were left, there seemed little doubt that the patient would speedily die from peritonitis, of which she had had several attacks, and from which she was apparently suffering in a subdued degree, or else that she would be worn out by the continued disturbance of digestion, and consequent impairment of nutrition, kept up by the foreign body. If, on the other hand, an attempt were made to remove it, there was every reason to apprehend that inflammation of the peritoneum might be lighted up, which would probably prove fatal in the patient's weakened state. We were, however, in hopes that the track along which the pencil lay had been shut off from the rest of the peritoneum by the deposit of lymph, and that thus the danger of peritonitis would be materially lessened. However, it appeared to us that the probable danger of extraction was less than the certain danger of allowing the pencil to remain in the abdominal cavity, and we accordingly came to the opinion that its removal ought to be attempted.

Operation.-At 4 p. m., the patient having been anæsthetized, the water drawn off, and the rectum ascertained to be empty, I proceeded to operate, being ably assisted by Mr. Bryant, who, passing his fingers deeply into the rectum, pushed the posterior end of the pencil upwards and forwards, so as to make the point project as much as possible. I cut down on this through the several layers of the anterior abdominal wall, until the fascia transversalis was reached, in which the black-lead point of the pencil was fixed, and through which it projected. I now slightly enlarged the opening in the fascia, and stripping back the tissues somewhat, exposed enough of the pencil to enable me to withdraw it with a pair of necrosis forceps. On removal it was found to have a strong feculent odour, and to be deeply stained in places with the intestinal contents. It was five and a half inches in length, cut to a sharp point, which was 
still perfect. The two halves of which it was composed had separated. On examining the pencil more closely, it was seen to be distinctly marked by three broad bands, which ran completely round it. Two of these, towards either extremity, were rough and dark with feculent staining; the third, in the centre, was smooth, clean, and quite free from any feculent imbibitions. From this examination it appeared as if the pencil had lodged in the interior of the intestine, and in contact with feculent matters in two situations. No feces or flatus, however, followed the extraction of the pencil, or escaped by the wound, which was closed by two sutures and some plaster.

The patient was put in bed with the knees raised, confined to ice- and barley-water, and opium was pretty freely given.

On the day following the operation, a severe attack of peritonitis came on, specially marked by incessant vomiting and retching, and by obstinate constipation. In spite of active treatment of the usual kind these symptoms continued, and the patient died on the fourth day after the operation.

Examination about fifty hours after death.-The body presented signs of commencing decomposition ; the abdomen was discoloured and tympanitic. On opening the peritoneal cavity, some flatus escaped. There were several ounces of turbid, rather dark-coloured serum in the cavity of the peritoneum, with flakes of recent lymph gluing the intestines together at several points. The omentum was much injected. About midway between the umbilicus and pubes, to the right of the mesial line, a mass of small intestine (ilium) was found glued together by old and tough lymph, forming a tumour composed of several coils of intestine, and altogether about as large as the fist. This mass was generally dark coloured, of a reddish purple tint, deeply injected, and presenting on its surface deposits of recent lymph in addition to the old lymph, by which its component coats were 
matted together. On attempting to separate the coats of which this mass was made up, some fluid feces and air bubbled out from between them, and on examining the source from which this came, it was found that two of the coils of intestine had been perforated, each in two places. In one, the apertures were close to each other, at the mesenteric border of the intestine. In the other, they were nearly opposite to one another, traversing the centre of the gut. These apertures were all rounded, smooth, and exactly of the same size and shape, corresponding in these respects to the pencil, by which they had been made. They were surrounded by a quantity of old and dense lymph, by which the neighbouring portions of intestine were so firmly attached to one another, and to the wounded gut, as to be separated with difficulty. The bladder, uterus, and rectum were sound.

On examining the vagina, a depressed cicatrix was found at its upper and posterior cul de sac, close by the side of the uterus. The peritoneal aspect of this cicatrix was thickened, puckered, and depressed.

From this it would appear that the pencil perforated the posterior wall of the vagina, passing upwards behind the bladder, and then traversing the peritoneal cavity. There can be little doubt that this accident happened whilst the patient, disturbed during her attempts to pass the foreign body into the urethra, allowed it to slip out of her grasp, and then suddenly sitting down, forced the pointed end of the pencil into the abdomen. She was seen and carefully examined a few hours afterwards by a medical practitioner of the town in which she was at the time residing, and he was unable to find any trace of the pencil, which he certainly would have done had it been lodging in the cavities of any of the pelvic organs. At this time, also, the intestine was doubtless perforated, and continued transfixed through two of its coils until the time of extraction, a period of nearly eight months. 
Not the least interesting feature of this case is the absence of all feculent extravasation into the cavity of the peritoneum, not only during the sojourn of the pencil within the abdomen, but after its removal. Whilst in the abdomen, and firmly fixed in the intestine which it traversed, the non-escape of feculent matters might be accounted for by the pencil, mechanically as it were, blocking up the apertures it had made; but after its withdrawal, the absence of extravasation must, I think, be accounted for by the influence of the uniform pressure of the abdominal organs on one another. 